\title{
Comments Supplemental Qualifiers Dataset
}

National Cancer Institute

\section{Source}

National Cancer Institute. Comments Supplemental Qualifiers Dataset. NCI Thesaurus. Code C147194.

A dataset containing supplemental information, specifically non-standard variables, to parent records in the comments domain. 